\begin{tabular}{lcr}
\hline \multicolumn{3}{c}{ A N N A L ES } \\
UNIVERSITATIS M A R I A E C URIE-SKŁ ODOWS K A \\
LOL. XXXVI & SECTIOFF & $1-2018$ \\
\hline
\end{tabular}

MONIKA BAZYL

ORCID ID: https://orcid.org/0000-0002-3857-171X

Uniwersytet Marii Curie-Skłodowskiej

\title{
La joie submerge mon coeur/La alegría inunda mi corazón. Dimension culturelle des métaphores de la joie en français et en espagnol
}

La joie submerge mon coeur/La alegría inunda mi corazón (radość wypełnia moje serce). Kulturowe uwarunkowania metafor konceptualnych radości w języku francuskim i hiszpańskim

La joie submerge mon coeur/La alegría inunda mi corazón. Cultural dimension of the metaphors of joy in French and Spanish

La linguistique cognitive fait partie de l'ensemble des domaines appelés «sciences cognitives» qui traitent des différentes fonctions de l'esprit et de la cognition humaine. Les autres disciplines constituant les sciences cognitives sont la psychologie, la philosophie, l'anthropologie, la neurobiologie et l'informatique. Une nouvelle étude du langage surgit dans les années 70, mais elle se développe considérablement à partir de 1987 lorsque deux ouvrages fondamentaux sont publiés.

L'apparition d'une nouvelle branche du langage crée une incompréhension entre les linguistes et les psychologues. Pour certains d'entre eux, la linguistique cognitive n'est pas une étude innovatrice parce que l'analyse du langage depuis une perspective similaire avait déjà été réalisée par la psychologie. C'est pour cette raison que, dans son article What's cognitivism about cognitive linguistic (1996), le psychologue Raymond W. Gibbs explique pourquoi le terme «cognitif» mérite la linguistique. Selon lui, la particularité de la nouvelle branche vient du fait qu'elle incorpore des trouvailles empiriques des autres disciplines dans la théorie linguistique. Grâce à la recherche continue de correspondances entre la pensée, l'expérience corporelle et le langage, la linguistique cognitive peut aboutir à des phénomènes totalement nouveaux, auparavant ignorés. 


\section{LES ÉMOTIONS ET LE LANGAGE : COMMENT DÉCRIT-ON LES ÉMOTIONS?}

Les émotions peuvent se refléter de trois façons dans le langage:

1. comme une description des émotions éprouvées par le sujet et d'autres personnes,

2. exprimées implicitement par le sujet,

3. exprimées involontairement par le sujet.

Zoltan Kövecses distingue deux types de langage des émotions: expressif, c'est-à-dire celui qui révèle l'état émotif du sujet à travers des interjections grâce au contexte partagé par l'auditeur, et descriptif - lorsque l'on utilise des substantifs et des adjectifs pour nommer les sentiments.

Élaborer une définition de l'émotion n'est pas une tâche simple, puisque c'est un phénomène psychique très complexe qui peut être traité sous un angle différent. Anna Wierzbicka (1971 : 30) affirme même qu’on ne peut définir les émotions :

Une émotion c'est quelque chose que l'on ressent, et pas quelque chose que l'on exprime avec des mots. Avec des mots, on peut exprimer des pensées, mais pas - nos émotions. La pensée est quelque chose qui possède une structure pouvant être reproduite à l'aide de mots. L'émotion, de par sa nature, est dépourvue d'une structure quelconque et, en conséquence, elle est inexprimable.

Pour décrire les émotions, il est indispensable de prendre en considération les étapes qui les accompagnent et qui se reflètent dans la communication verbale: le type de cause, de sensation ressentie par le sujet, la manière d'évaluer la stimulation, la manière de manifester l'émotion (mouvements, gestes, mimiques, réactions physiologiques et leur association avec des manifestations verbales).

Selon Iwona Nowakowska-Kempna (1995), la description linguistique des émotions a pour objectif de:

- dénommer les émotions, le comportement qui cause l'émotion et les sentiments d'une personne;

- dénommer la manifestation des émotions figées dans le sens des phraséologismes;

- dénommer le comportement lié aux émotions.

Après une brève présentation des principes théoriques de la linguistique cognitive, nous passons à l'analyse détaillée de la conceptualisation de la joie en français. Notre étude sera basée sur la théorie de la métaphore de George Lakoff et Marc Johnson (1980). La métaphore est présente dans la vie quotidienne et touche tous les aspects de notre vie. Les linguistes américains montrent qu'elle imprègne la vie quotidienne, pas seulement le langage, mais aussi la pensée et l'action. C'est un moyen cognitif fondamental qui sert à conceptualiser différents domaines. 


\title{
LES MÉTAPHORES CONCEPTUELLES DE LA JOIE
}

\section{La métaphore de la substance}

Pour décrire les métaphores les plus caractéristiques de la joie, nous avons choisi les métaphores ontologiques qui présentent la joie comme une substance quantifiable :

\author{
LA JOIE EST UN LIQUIDE: \\ une fontaine de joie \\ une vague de joie \\ un flot de joie \\ $X$ est submergé, inondé de joie \\ la joie submerge, inonde $X$ \\ les larmes de joie \\ $X$ nage dans la joie
}

es. manantial/ fuente de alegria

es. onda/ oleada/ ola de alegría

es. X está inundado de alegría

es. la alegría inunda mi corazón / una súbita alegría inunda X es. gota de alegría

La conceptualisation aquatique révèle la profondeur de la joie. Les mouvements de l'eau marine (une vague, un flot de joie) peuvent être comparés à des excès de joie. Le sens de l'expression métaphorique quelqu'un est submergé / inondé de joie évoque la puissance de l'eau en tant qu'élément naturel. Selon RAE (Real Academia Española), le verbe submerger signifie 'recouvrir complètement, inonder' inundar - 1. 'llenar o cubrir un lugar de agua u otro líquido, 2. 'dicho de una emoción o de un sentimiento: embargar a alguien'. Celui qui éprouve de la joie est totalement recouvert, inondé. Cette image associée à la joie sert à exprimer l'intensité maximale de l'émotion. Le sujet est totalement sous l'influence de la joie, des pieds jusqu'à la tête. La même expression existe en espagnol: X está inundado de alegría, una súbita alegría inunda $X$.

Le verbe déferler désigne le fait de se répandre avec impétuosité (en parlant d'un phénomène). Au sens figuré, il sert à exprimer une émotion qui survient brutalement, avec une force envahissante (une vague de joie déferle sur quelque chose, la colère déferle, une vague d'un chagrin intense déferle en elle).

En revanche, la métaphore nager dans la joie évoque l'image d'un homme tranquille et joyeux, profitant du moment de joie.

Au sens propre, manantial et fuente désignent un flot sortant de la terre ou de la roche d'eau d'une manière naturelle. L'expression un manantial de alegría ('source de joie') décrit la phase initiale de l'état (quelqu'un commence à être joyeux).

Dans les langues espagnole et française, la joie est conceptualisée en tant qu'un liquide (une vague, onda) qui submerge l'expérienceur ou remplit son intérieur. Dans ce dernier cas, la personne qui éprouve cette émotion est représentée comme un récipient rempli d'un liquide. 
Ola et oleada traduisent un mouvement de l'eau sur la surface de la mer ou dans un lac, mais un sens figuré implique une grande quantité ou l'excès de quelque chose qui surgit d'une manière subite. Il semble intéressant de remarquer que ces termes apparaissent fréquemment avec le verbe inundar (fr. inonder) pour, dans son sens littéral, transmettre l'idée de couvrir un lieu ou une personne d'un liquide. La métaphore sert à exprimer l'intensité maximale de l'émotion. En revanche, oleada dénote quelque chose d'impossible à contrôler et hostile pour l'être humain.

\section{LA JOIE EST UN CORPS GAZEUX: \\ le cour gonflé de joie}

Dans ce cas-là, la joie est présentée comme un corps gazeux qui remplit le récipient (le cœur). Le verbe gonfler, signifiant 'rendre (plus) ample ou augmenter le volume', donne l'impression que notre corps devient plus ample que d'habitude.

La métaphore ontologique de la substance suppose aussi la possibilité de présenter l'émotion comme une entité quantifiable:

\begin{tabular}{|c|c|}
\hline une dose de joie & es. dosis de alegría \\
\hline beaucoup del peu del tant de joie & es. mucha/ poca/ demasiada/ bastante/ tanta \\
\hline grande joie & es. gran/ inmensa/ enorme/ mayor alegría \\
\hline \multicolumn{2}{|l|}{ petite joie } \\
\hline une joie excessive & es. excesiva alegría \\
\hline une joie mesurée & es. mesurada alegría \\
\hline \multirow[t]{2}{*}{ une injection de joie } & es. inyección de alegría \\
\hline & es. mesurada alegría (fr. la joie mesurée) \\
\hline
\end{tabular}

En français et en espagnol, les expressions une dose de joie et une injection de joie, dosis de alegría projettent l'image de joie comme si elle était une substance quantifiable pour une portion déterminée. La joie peut être aussi mesurée de façon approximative (beaucoup, un peu de, micho, poco). Ces exemples prouvent que l'esprit humain conçoit le concept abstrait de la joie comme une matière quantifiable: beaucoup et grand désignent des quantités très importantes alors que leur antonyme petit - une taille inférieure à la moyenne.

Il faut noter qu'en français et espagnol, les collocations une joie mesurée, mesurada alegría servent à exprimer à la fois une intensité faible de l'émotion (retenue, modérée) et un jugement de valeur: «Con mesurada alegría se celebra triunfo en el Este) ${ }^{1} »$; «Joie mesurée, surprise et relativisme: réactions variées chez les Rochelais $»^{2}$.

${ }^{1} \mathrm{http} / / / \mathrm{www} \cdot a b c . c o m . p y / d e p o r t e s / c o n-m e s u r a d a-a l e g r i a-s e-c e l e b r a-t r i u n f o-e n-e l-e s t e-$ 826941.html; consulté le 23.02.2018.

${ }^{2}$ https://www.rugbyrama.fr/rugby/xv-de-france-les-rochelais-de-la-joie-mesuree-a-la-joietout-court_sto6382085/story.shtml; consulté le 23.02.2018. 


\title{
2. La métaphore du contenant ou du contenu
}

Dans bon nombre d'expressions, le corps (ou l'individu) est comme un « contenant» de l'émotion (celle-ci étant conceptualisée comme «contenu») :

\author{
LA JOIE EST UN CONTENU: \\ $X$ est rempli de joie \\ $X$ est plein de joie \\ le cœur de X est plein de joie \\ $X$ contient sa joie \\ $X$ comble de joie
}

es. estar uno repleto/ pleno/ pletórico de alegría es. estar uno lleno de alegría

es. corazón de X està lleno de alegría

es. tener uno alegría

Les verbes utilisés ont un sens figuré et servent à exprimer le degré d'intensité de l'émotion:

Remplir 1. 'mettre quelque chose en assez grande quantité dans un contenant, le rendre plein' 2. pénétrer quelqu'un d'un sentiment, d'une impression'.

Combler 1. 'remplir un creux, un vide, une cavité d'un matériau solide de manière à ce qu'il n'y ait plus de creux, que la cavité soit pleine' 2. 'satisfaire pleinement, réaliser le souhait, le désir de quelqu'un'.

Les expressions métaphoriques basées sur le modèle de possession sont généralisées dans la langue espagnole pour exprimer certaines sensations physiques (p. ex. la soif, le froid, etc.) et les états animiques (p. ex. la peur). Elles sont de ce fait si souvent utilisées et systématiques que leur caractère métaphorique est à peine perceptible.

Dans ces deux langues, l'expérienceur est présenté comme un contenant de l'émotion.

La métaphore du trop-plein est motivée par l'image d'un liquide qui monte jusqu'à ce que le récipient explose en expulsant ce qu'il contenait:

\section{LA JOIE EST UN CONTENU QUI DÉBORDE LE CONTENANT: \\ une joie expansive, débordante \\ $X$ déborde de joie \\ X s'évanouit de joie \\ $X$ explose de joie \\ es. tener una alegría desbordante \\ es. llenarse/ colmarse uno de alegría \\ es. X desborda de alegría \\ es. desmayarse de la alegría \\ es. estallar/ reventar uno de alegría \\ (registre vulgaire)}

une joie qui éclate sur le visage de $X$ une éruption de joie es. explosión de alegría

Le verbe déborder fait une claire référence à l'action d'extérioriser et véhicule l'idée de dépasser les limites fixées. Alors qu'exploser signifie 'se désintégrer, sous l'action d'un mélange détonant, de manière violente et instantanée, généralement 
avec bruit et, parfois, en projetant des éclats'. Par rapport au substantif éruption, il signifie une sortie brusque d'une chose hors de corps. Les constructions qui comportent ces verbes décrivent une émotion qu'on ne peut pas maîtriser.

\section{La métaphore de l'objet}

La joie peut être conceptualisée en termes d'objet. Les exemples ci-dessous illustrent bien ce type de conceptualisation.

\section{LA JOIE EST UN OBJET:}

$X$ a la joie de faire quelque chose

$X$ partage la joie de quelqu'un

$X$ prend part à la joie de quelqu'un

es. estar uno poseído por alegría

es. arrebatar uno alegría a alguien (fr. arracher) es. proporcionar uno alegría (fr.proportionner) es. recibir uno alegría (fr. recevoir) es. perder uno alegría (fr. perdre)

Il est intéressant de remarquer que la joie apparaît ici comme une émotion qu'on partage avec quelqu'un. En espagnol, c'est la joie qui possède quelqu'un.

En espagnol, il existe une possibilité d'arracher, proportionner, recevoir et perdre la joie. Selon RAE, arrabatar signifie 'enlever avec violence et force', proporcionar - 'disposer et ordonner quelque chose en proportion', recibir - 'admettre, accepter, approuver quelque chose' et perder - 'se voir dépossédé d'un bien'.

\section{La métaphore de l'organisme vivant}

La joie peut être perçue comme un organisme vivant. La métaphore LA JOIE EST UN ÊTRE VIVANT a un caractère général dont dérive la métaphore spécifique: LA JOIE EST UNE PLANTE.

LA JOIE EST UN ÊTRE VIVANT:

une joie de vivre

une joie naît/renaît

une joie saisit $X$

une joie vient/revient

LA JOIE EST UNE PLANTE:

une joie qui fait fleurir

une vie fleurit de joie

$X$ laisse fleurir la joie es. alegría de vivir

es. la alegría reina/ impera

es. morir(se) de alegría (fr. mourir)

es. la alegría brota

es. exuberante alegría

es. alegría florida 
Nous voyons en examinant les exemples que la pensée humaine se sert des concepts proches de notre expérience, des termes de la nature. Déjà Lakoff et Johnson ont démontré que l'être humain conceptualise les idées abstraites en termes plus concrets.

En français et en espagnol, le verbe naître souligne le moment auquel l'événement commence à se produire avec indépendance. En revanche, vivre et vivir présente le trait caractéristique de l'être vivant. Le concept de joie de vivre désigne un sentiment de bien-être général, de bonheur complet qui vient du simple fait d'exister.

Le verbe fleurir dans sa signification littérale implique quelque chose qui a des fleurs et exubérant dénote quelque chose «produit en extrême abondance».

En espagnol, l'expression métaphorique morir(se) de alegría contient le verbe qui sert à intensifier le nom qui se rapporte à l'émotion.

\section{La métaphore de la clarté}

Comme nous l'avons déjà vu, l'homme conceptualise les émotions en termes proches de son expérience. Ainsi, pour conceptualiser la joie, on fait référence à la perception visuelle - à la lumière.

\author{
LA JOIE EST UNE CLARTÉ: \\ une joie rayonnante \\ une joie vive \\ $X$ brille de joie \\ $X$ rayonne de joie \\ des yeux pétillant de joie \\ des yeux qui brillent de joie
}

\author{
es. estar uno radiante de alegría \\ es. irradiar/lucir/ emanar uno alegría \\ es. iluminarse la cara a alguien \\ es. tener uno el brillo de alegría en los ojos
}

La personne qui éprouve de la joie est décrite comme si elle était l'origine de la lumière qui sort de son intérieur. Comme les émotions se reconnaissent généralement à partir de l'expression du visage, en particulier à travers les yeux qui ont été reconnus depuis toujours comme «le reflet de l'âme», on trouve des expressions qui sont motivées par la luminosité des yeux.

Selon RAE, les verbes espagnols irradiar, lucir, emanar sont synonymes des verbes briller, illuminer.

\section{La métaphore de la maladie}

Il est un fait connu que la joie appartient à l'inventaire des émotions positives, mais quand elle devient trop intense, elle peut causer toute une série de dérangements dans la santé. Comme le signale Kövecses, la métaphore de maladie est une 
spécification du modèle de la force. Cela dit, plusieurs expressions présentées dans cet article ont une base métonymique, puisque, comme il a été précisé auparavant, cette émotion peut provoquer des réactions incontrôlées liées à la libération de dopamine.

LA JOIE EST UNE MALADIE:

un délire de joie

une joie délirante

$X$ est malade de joie es. estar uno enfermo de alegría

es. contagiarse uno de alegría (fr. contaminer)

es. transmitir uno alegría

es. la alegría contagiosa

Selon Larousse, délirant désigne un état accompagné de délire ou la manifestation d'une grande excitation. Dans le langage familier, l'adjectif renvoie à un état qui dépasse les limites raisonnables.

Les métaphores espagnoles méritent notre attention. Les verbes transmitir, contagiar sont associés à des connotations liées à une maladie qui peut être contagieuse. En français, un symptôme de la maladie est un délire. Au sens figuré, l'expression française révèle qu'à cause de la joie, nous pouvons voir des choses irrationnelles, tandis que l'expression espagnole indique quant à elle qu'au contact de gens heureux, nous pouvons nous aussi devenir joyeux.

\section{LES MÉTONYMIES DE LA JOIE}

La métonymie s'explique par une ellipse. On recourt tout naturellement au mécanisme métonymique pour pallier aux insuffisances de vocabulaire. Si un objet n'a pas de nom dans le langage, on pourra le désigner par une périphrase plus au moins étendue. La rhétorique traditionnelle avait remarqué que la plupart des catachrèses font appel au mécanisme métonymique.

La conceptualisation des émotions n'est pas seulement basée sur les métaphores. La métonymie y joue aussi un rôle important. Elle est basée sur le principe L'EFFET D'UNE ÉMOTION POUR L'ÉMOTION. Ce modèle est réalisé par les expressions qui dénotent les manifestations externes de la joie.

\section{LES MÉTONYMIES RENVOYANT À LA MANIFESTATION EXTERNE DE LA JOIE}

D'abord, nous avons constaté qu'une quantité considérable d'expressions dénotent le comportement d'une personne joyeuse. Les manifestations externes 
(physiques) de la joie sont souvent marquées de nuances culturelles, ce qui trouve son reflet dans le langage.

\author{
un cri de joie \\ $X$ crie de joie \\ $X$ bondit de joie \\ $X$ gambade de joie \\ $X$ saute de joie
}

\author{
es. gritos de alegría \\ es. gritar de alegría, chillar uno de alegría \\ es. brincar uno de alegría/ dar uno brincos de alegría \\ es. saltar uno de alegría/ dar uno saltos de alegría
}

En français et en espagnol, les collocations exprimant la joie contiennent les verbes de mouvement qui désignent une série de bonds : sauter/saltar, bondir/brincar, gambader. Le verbe gambader suggère de sa nature un mouvement joyeux, folâtre. Bondir sert à décrire un mouvement brusque en l'air. L'accent est mis sur l'idée d'agilité, de souplesse.

D'autres expressions comportent les collocatifs qui expriment des manifestations vocales, sans communiquer véritablement de contenu, par exemple crier de joie, cris de joie, gritos de alegría, exlamaciones de alegría.

Quant aux collocations X frétille de joie, X tiembla de alegría, elles dénotent une manifestation physique réactive non contrôlée par l'expérienceur.

La joie peut être vécue et considérée dans ses expressions les plus spectaculaires et le plus souvent collectives:

\author{
un chant de joie \\ une danse de joie \\ $X$ chantel chantonne de joie \\ es. danza de alegría \\ es. cantar de alegría \\ $X$ danse de joie \\ es. bailar de alegría \\ $X$ trépigne de joie \\ $X$ tape des mains, X applaudit de joie \\ es.batir palmas de alegría
}

En français et en espagnol, pour manifester la joie, on peut batir palmas de alegría ou taper des mains lors d'une fête.

La joie et la alegría s'expriment aussi à travers d'autres réactions possibles corporelles: les larmes, le troublement ou la luminosité des yeux.

Les manifestations externes qui peuvent être observées chez une personne joyeuse permettent d'identifier cette émotion.

\author{
LE CHANGEMENT DE L'ASPECT DU VISAGE \\ une joie éclate sur son visage \\ es. iluminarse la cara a alguien \\ un sourire de joie \\ es. sonrisa de alegría \\ des yeux qui brillent de joie \\ es. tener uno el brillo de alegría en los ojos
}

des yeux qui pétillent de joie

un visage qui exprime la joie 
$X$ brille de joie

des larmes de joie

des pleurs de joie

X pleure de joie es. estar radiante de alegría

es. lágrimas de alegría

es. llorar uno de alegría, sollozar uno de alegría

En France et en Espagne, la joie est une émotion très facile à reconnaître, puisque le sourire l'accompagne normalement. Cette mimique est associée par convention à la joie. Mais la joie peut être aussi accompagnée de pleurs qui normalement sont des symptômes de tristesse et de douleur.

\section{CONCLUSION}

Notre étude permet de montrer qu'en français et en espagnol, on compte un grand nombre de métaphores et de métonymies qui renvoient à la joie. On note que la plupart d'entre elles sont les mêmes, par exemple celles qui présentent la joie comme une clarté ou un être vivant. Dans les deux langues comparées, la joie est vue aussi comme une maladie, mais ses symptômes sont différents. En français, celui qui éprouve de la joie, peut être amené à avoir des délires et se comporter de ce fait d'une manière irrationnelle. En revanche, en espagnol, la joie se communique d'une personne à l'autre, se transmet par la fréquentation intense (alegría contagiosa).

En ce qui concerne les expressions métonymiques, elles renvoient aux mêmes manifestations physiques externes qui impliquent ou non une certaine activité, et aussi une certaine maitrise (ou non) de la joie.

\section{RÉFÉRENCES BIBLIOGRAPHIQUES}

Cosnier, J. (1994). Psychologie des émotions et des sentiments. Paris: Retz.

Dobrzyńska, T. (1943). Metafora. Wrocław: Zakład Narodowy im. Ossolińskich, Wydawnictwo Polskiej Akademii Nauk.

Fernández-Abáscal, E. (1997). Psicología General. Motivación y Emoción, Editorial Centro de Estudios Ramón Areces. Madrid: Editorial Universitaria Ramón Areces.

Fortin, B. (2002). La gestion des émotions. Montréal: Édistions CPF.

Henry, A. (1971). Métaphore et Métonymie. Paris : Éditions Klincksieck.

Kövecses, Z. (2003). Metaphor and Emotion: Language, Culture and Body in Human Feeling. Cambridge: Cambridge University Press.

Krzyżanowska, A. (2011). Aspects lexicaux et sémantiques de la description des noms d'affect en français et en polonais. Lublin: Wydawnictwo Uniwersytetu Marii Curie-Skłodowskiej.

Johnson, M., Lakoff, G. (1985). Les métaphores dans la vie quotidienne. Paris: Les Éditions de Minuit. 
Le Guern, M. (1973). Sémantique de la métaphore et de la métonymie. Paris: Librairie Larousse. Mikołajczuk, A. (2009). Obraz radości we współczesnej polszczyźnie. Warszawa: Wydawnictwo Naukowe Semper.

Świątek, J. (1998). W świecie powszechnej metafory. Metafora językowa. Kraków: Polska Akademia Nauk.

Tabakowska, E. (2001). Językoznawstwo kognitywne a poetyka przekładu. Kraków: Universitas. Wierzbicka, A. (1971). Kocha, lubi, szanuje. Medytacje semantyczne. Warszawa: Wiedza Powszechna.

\section{RÉSUMÉ}

Cet article a pour objectif de décrire les ressemblances et les différences dans la conceptualisation de la joie en français et celle de alegría en espagnol. Dans notre recherche, nous nous appuyons sur la théorie de la métaphore proposée par G. Lakoff et M. Johnson. Dans la linguistique cognitive, la métaphore est un des outils les plus importants pour essayer de comprendre le domaine abstrait de notre expérience.

D'abord, nous étudions la joie vue comme un concept psychologique pour passer ensuite à la description d'une série de métaphores conceptuelles et de métonymies de la joie. Les exemples analysés sont tirés de la presse et d'œuvres littéraires françaises et espagnoles.

Notre analyse montre que les expressions métaphoriques communes pour le français et l'espagnol sont celles, qui représentent la joie comme une lumière, un organisme vivant ou une maladie. D'autre part, les différences concernent, par exemple, les conséquences résultant de l'état pathologique. Ainsi, les Français qui éprouvent une grande joie peuvent se comporter de manière irrationnelle. En revanche, en espagnol, la joie est représentée comme une maladie contagieuse, c'est-à-dire une émotion qui se communique d'une personne à l'autre.

Mots-clés : linguistique cognitive, métaphore conceptuelle, conceptualisation de la joie et de la alegría

\section{ABSTRACT}

The purpose of this article is to offer a description of how to conceptualize the joy in French and Spanish. The analysis is based on the concept of a metaphor developed by George Lakoff and Mark Johnson. In the assumptions of cognitive linguistics, metaphor is the main and the most important carrier of thinking and understanding.

In the initial part of this article, in order to reflect on the readable image of joy completely, it was considered important to discuss it within the linguistics and psychological perspectives. The analysis reveals that there are a number of expressions that indicate the initial moment of joy. This feeling may appear gradually or occur suddenly. However, most psychologists describe the joy as a dynamic sensation as a result of stimulus.

Our analysis shows that metaphorical expressions are common for French and Spanish, which represent joy as a light, a living organism or disease. On the other hand, the differences concern, for example, the consequences resulting from the disease state. In French, a subject experiencing emotions of joy can seem or behave irrationally. In contrast, in Spanish, joy is seen as a contagious disease.

Keywords: cognitive linguistics, conceptual metaphor, conceptualization of joy and alegría 


\begin{abstract}
ABSTRAKT
Celem niniejszej pracy jest opis sposobu konceptualizacji radości w języku francuskim i hiszpańskim. Analiza opiera się na koncepcji metafory opracowanej przez George’a Lakoffa i Marka Johnsona. W ujęciu językoznawstwa kognitywnego metafora jest najważniejszym narzędziem poznania i rozumienia pojęć abstrakcyjnych.

W części wstępnej artykułu, w celu ukazania w pełni czytelnego obrazu radości, emocja ta została przedstawiona z perspektywy językowej i psychologicznej. Istnieje wiele zwrotów i wyrażeń, które wskazują na początkowy moment radości. Uczucie to może pojawiać się stopniowo bądź wystąpić nagle. Jednakże większość psychologów opisuje radość jako uczucie dynamiczne, występujące na skutek bodźca.

Analiza pokazuje, że wspólne dla języka francuskiego i hiszpańskiego są wyrażenia metaforyczne, które przedstawiają radość jako światło, żywy organizm lub chorobę. Natomiast różnice dotyczą na przykład następstw wynikających ze stanu chorobowego. W języku francuskim podmiot doznający emocji radości może majaczyć lub zachowywać się irracjonalnie. Natomiast w języku hiszpańskim radość jest postrzegana jako zaraźliwa choroba.
\end{abstract}

Słowa kluczowe: językoznawstwo kognitywne, metafora konceptualna, konceptualizacja radości 